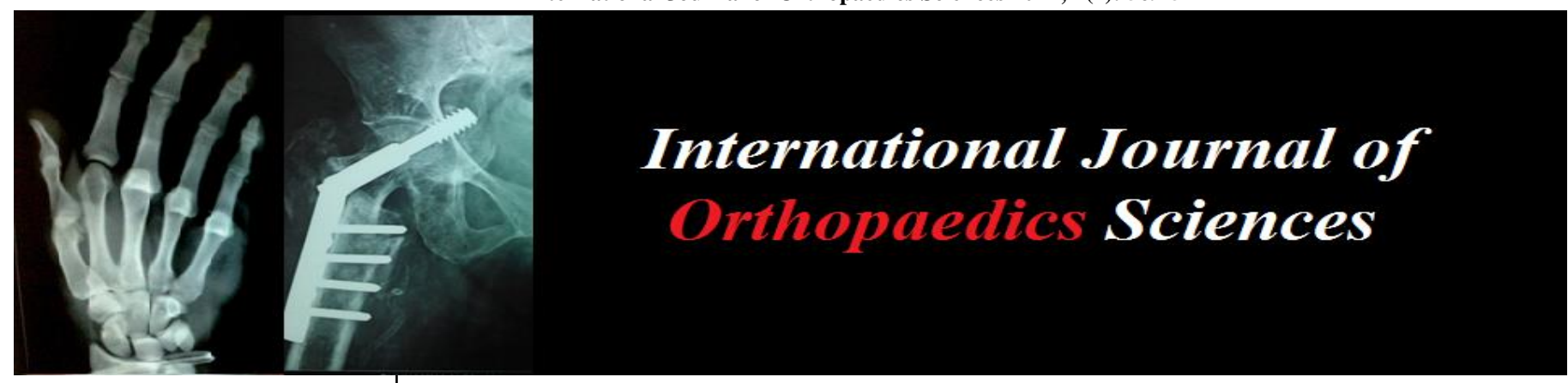

E-ISSN: 2395-1958

P-ISSN: 2706-6630

IJOS 2021; 7(4): 569-577

(C) 2021 IJOS

www.orthopaper.com

Received: 28-08-2021

Accepted: 30-09-2021

Dr. Sadda Sarath Reddy Postgraduate Resident,

Department of Orthopaedics,

Narayana medical college and

hospital, Chinthareddypalem,

Nellore, Andhra Pradesh, India

Dr. Konda Siva Sankar Reddy Assistant Professor, Department of Orthopaedics, Narayana medical college and hospital, Chinthareddypalem, Nellore, Andhra Pradesh, India
Corresponding Author: Dr. Konda Siva Sankar Reddy Assistant Professor, Department of Orthopaedics, Narayana medical college and hospital, Chinthareddypalem, Nellore, Andhra Pradesh, India

\section{Functional and radiological outcome of closed talus neck fractures managed by open reduction and internal fixation: A case series study}

\author{
Dr. Sadda Sarath Reddy and Dr. Konda Siva Sankar Reddy
}

DOI: https://doi.org/10.22271/ortho.2021.v7.i4h.2936

\section{Abstract}

Background and Objectives: Displaced talar fractures remain a therapeutic challenge for orthopaedic surgeons. According to the literature, these fractures are often associated with high complication rates, including malunion, osteonecrosis, osteoarthritis. The operative treatment of such fractures seems to require a balance between an aggressive treatment with a strict anatomical reduction and essential respect of soft tissues to limit skin complications or osteonecrosis. Fractures of the talus are known to be relatively uncommon. Talus fractures comprise approximately 0.1 to 0.85 percent of all fractures. Most occur as a result of high-energy trauma, such as motor vehicle accidents. As a result, talus fractures are often accompanied by other injuries, including dislocation of adjacent joints and fracture of neighboring bones. However, due to the relatively better diagnostic methods introduced more talar fractures have been recognized and it remains the second most common tarsal bone to get fractured after calcaneum.

Methods: 30 patients were selected in an age group of 15-60 years who suffered from closed talus fractures either by road traffic accidents or fall injuries. After clinical evaluation and investigations, Xray and CT scan of ankle of patients were done. The patients underwent operative fixation of talus fracture by cancellous screw fixation and post-operative immobilization was given. Following discharge, patients were followed up at 3rd month, 6th month, 1 year, and during each follow up serial Xrays were taken anteroposterior, lateral, mortise view of the ankle joint. Clinical assessment of the ankle was done using Baird Jackson score, Olerud Molander, AOFAS scoring.

Results: 10 of the 30 cases had signs of avascular necrosis in follow up Xrays taken and there were clinical complaints of pain and inability to walk and undergo daily activities along with gait abnormalities.8 out of these 10 cases had joint incongruity and ankle joint pain and restriction of movement signifying arthritic changes. 2 cases had non-union seen at 3rd month post-op Xray and were advised and put on below-knee cast and immobilization for 8 weeks. In all cases, cancellous screws were used as the implant of choice and no implant-related complications were found in any of the patients. There was no post-operative skin infection or skin necrosis seen in any patients.

Conclusion: Open reduction and internal fixation are recommended for the treatment of displaced talar neck and/or body fractures. A delay in surgical fixation does not appear to affect the outcome, union, or prevalence of osteonecrosis, rather the type of fractures that might be indirectly influenced by the mechanism of injury provides a statistical significance.

Keywords: talus, talus neck fracture, cancellous screw, Baird Jackson score, Olereud Mollander score, AOFAS score, osteonecrosis

\section{Introduction}

Fractures of the talus are known to be relatively uncommon. Talus fractures comprise approximately 0.1 to 0.85 percent of all fractures. Most occur as a result of high-energy trauma, such as motor vehicle accidents. As a result, talus fractures are often accompanied by other injuries, including dislocation of adjacent joints and fracture of neighboring bones. However, due to the relatively better diagnostic methods introduced more talar fractures have been recognized and it remains the second most common tarsal bone to get fractured after calcaneum. Displaced fractures of the talar neck and body are best treated urgently, although the exact timing is controversial. Reduction of dislocated joints is critical to maintain vascularity to the talar body where possible and to reduce tension on the soft tissues and neurovascular structures around the foot and ankle. 
Early reduction is theorized to assist the restoration of blood flow and potentially decrease the rate of osteonecrosis. However, clinical studies have, to date, not demonstrated a significant effect of surgical timing on rates of osteonecrosis. When delayed treatment is an unfortunate necessity due to the condition of the patient, a prolonged delay in transfer, or other reasons, immediate management should still include an attempt at reduction of dislocations. Preferred surgical timing for talar neck fractures is controversial.

A recent survey of orthopaedic trauma experts regarding the timing of surgical treatment for displaced talar neck fractures revealed that $40 \%$ of respondents felt treatment should occur within 8 hours, and 36\% felt treatment should occur within 24 hours; the remaining $24 \%$ felt treatment after 24 hours was acceptable. The principles of surgery are to obtain an anatomic reduction of the talar neck fracture and the associated joints and to achieve sufficient stabilization to facilitate early motion. A variety of surgical techniques have been described to accomplish these principles. Emergent reduction of dislocated joints, precise fracture reduction and stabilization, and protection of the remaining vascular supply and soft tissue envelope provide the best probability of regaining an excellent functional result. Each talar body injury is different and requires individual preoperative planning to achieve an open reduction and then stable fixation. Screws alone or plates and screws can each be used. Occasionally, lag screws alone, if the fragments are large, will suffice in securing stable fixation of fractures of the body. Fractures of the talus comprises of three basic types- Talar head, Talar neck, Talar dome and body and transchondral fractures. Fractures of the lateral and medial processes of the talus occur in trivial injuries like eversion and inversion injuries.

Talar neck fractures are classified according to Hawkins classification.

A) Type I- Non displaced talar neck fracture

B) Type II-Displaced with subluxation or dislocation of subtalar joint

C) Type III-Displaced with dislocation of the body from ankle mortise

D) Type IV-Dislocation of subtalar joint with subluxation or dislocation of the talonavicular joint.

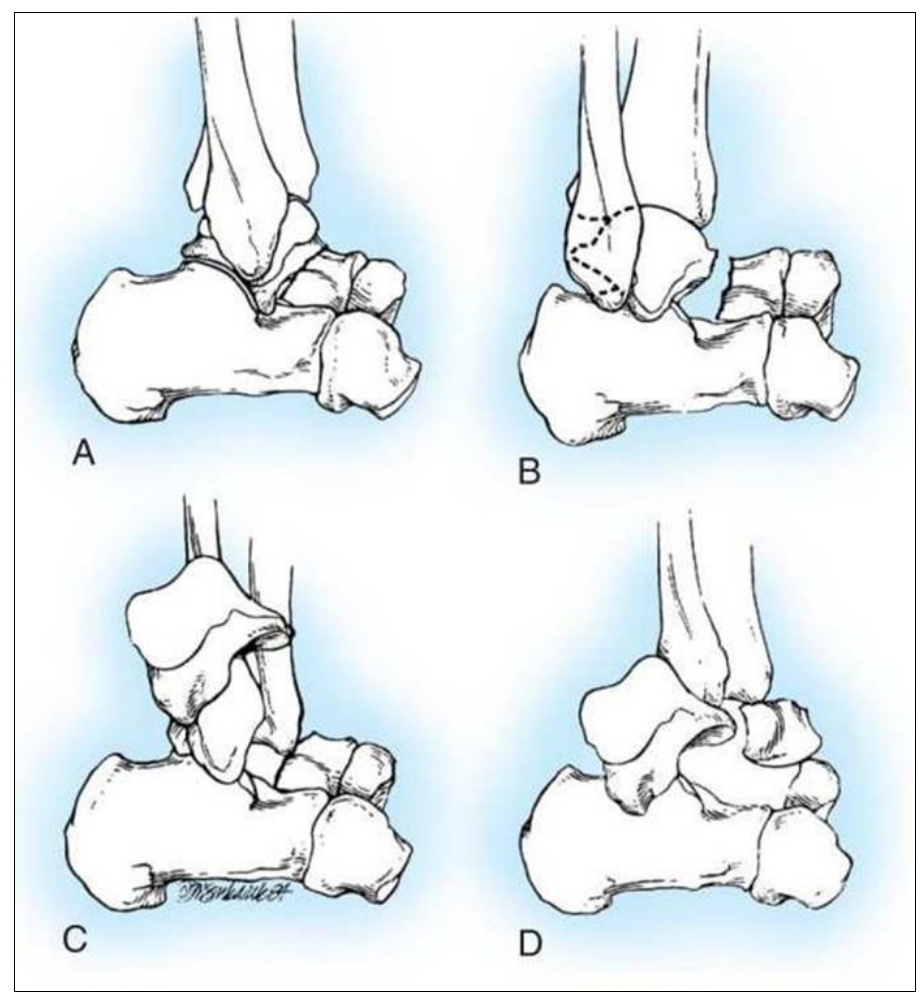

Talar neck fractures are classified according to Hawkins classification

\section{Aim \& Objectives}

1. To assess the functional and radiological outcome of closed talus neck fractures managed by open reduction and internal fixation.

2. To assess complications associated.

\section{Materials and Methods}

Place of study: Department of Orthopaedics, Narayana medical college and hospital, Andhra Pradesh.

Period of study - for a period of 24 months from June 2017 to June 2019

\section{Inclusion criteria}

Closed fracture of the talus with or without dislocation

- $\quad$ Road traffic accidents and fall injury
- $\quad$ Age - $15-60$ years

\section{Exclusion criteria}

- $\quad$ Compound fractures with Talus bone exposed

- Neglected fractures with > 3 months duration

- $\quad$ Prior native treatment done

\section{Sample size: 30}

\section{Pre-operative evaluation}

Detailed clinical examination

Complete Haemogram

Radiographs of the ankle and foot- anteroposterior and lateral views CT ankle with $3 \mathrm{D}$ reconstruction 


\section{Implants used}

In open reduction and internal fixation of the talus, $4 \mathrm{~mm}$ partially threaded cancellous screws were used after preliminary reduction with $\mathrm{K}$ wires.

Choice of approach- Anterolateral approach was most commonly employed followed by anteromedial approach and in cases where subtalar involvement was appreciated, a combined anteromedial and anterolateral approach was done.

\section{Post-Operative protocol}

Post-operatively intravenous antibiotics were administered for 5 days and oral antibiotics for 7 days. Suture removal was done on 12th post-operative day. Mobilization of ankle was started on day 1 postoperative day was divided into three phases as follows:

\section{Phase I week 1-6}

A) Surgical scar protection

B) Mobilization of ankle/foot to increase joint mobility

C) Active ROM exercises (i.e. ankle pumps) to increase circulation to the foot and promote cartilage healing

D) Instruction in non-weight bearing crutch ambulation

\section{Phase II week 6-12}

Initiate instruction in partial weight-bearing restriction with crutch ambulation.

Pain-free open chain exercises with the band.

\section{Phase III- week 12-24}

Progressive resistive strengthening of ankle musculature with band balance

Gait training on the treadmill with progression to incline surface

Single leg support activities

Fast walking with progression to jogging for patient-specific activities

\section{Follow Up}

Patients were followed up at first month (4weeks), third month (12 weeks), and 6 months. During follow-up, X ray radiograph of the ankle joint was taken in both anteroposterior view and lateral view and mortise view to assess the reduction as well as to analyze and check for any signs of avascular necrosis and also to see joint congruity. Clinical evaluation included range of motion of the ankle, complaints of pain and tenderness or swelling in the ankle joint. A gait examination was done. Implant assessment- the Screw position was assessed radiologically.

\section{Results}

A total of 34 patients are included in the study, of which we lost follow-up for 4 patients. The follow-up period ranged from 6 months to 18 months. Results are analyzed both clinically and radiologically. Time of clinical union is defined as the period between operation and full weight-bearing without external support along with radiologically healed fracture out of the 30 patients, the majority are from the 15-30 years age group i.e, 14 patients followed by 10 patients from 31-45 years age group, 6 patients from 46-60 years age group, with a mean age of $33.9 \pm 14.93$ years. The majority affected are males ( 22 patients) compared to females ( 8 patients). The commonest mode of injury falling from height accounting for 18 patients followed by road traffic accidents in 12 patients. 10 patients have associated fractures like malleolus fracture in 6 patients ( 4 medial malleolus and 2 bimalleolar), calcaneal fracture in 2 patients, tibia shaft fracture in 2 patients. Among these, 6 are Hawkins type I, 12 are Hawkins type II, 8 are Hawkins type III and 4 are Hawkins type IV.

All the fractures are managed by internal fixation using $4 \mathrm{~mm}$ partially threaded cancellous screws using an anterolateral approach in 14 patients, anteromedial approach in 12 patients, anterior approach in 2 patients, and dual approach (anteromedial and anterolateral) in 2 patients. Mean time elapsed from the day of injury to the day of surgery is $7.53 \pm$ 7.41.

Functional outcome is assessed using Baird Jackson scoring system with a mean score of $62.48 \pm 16.67$, with the majority having poor outcomes i.e, 26 patients, fair in 4 patients, good in 4 patients. AOFAS mean score of $58.53 \pm 13.85$, with the majority having fair outcome i.e, 12 patients, poor in 10 patients, good in 6 patients, and excellent in 2 patients. Olerud and Molander mean score of $53.9 \pm 17.72$, with the majority having fair outcome i.e, 18 patients, good in 8 patients, and poor in 4 patients.

Complications: In our study, 10 cases had signs of avascular necrosis in follow-up Xrays taken and there were clinical complaints of pain and inability to walk and undergo daily activities along with gait abnormalities. 8 cases had joint incongruity and ankle joint pain and restriction of movement signifying arthritic changes. 2 cases had non-union seen at 3rd month post-op Xray and was advised and put on Below-knee cast and immobilization for 8 weeks. In all cases, cancellous screws were used as the implant of choice and no implantrelated complications were found in any of the patients. There was no postoperative skin infection or skin necrosis seen in any patients.

Table 1: Age distribution of patients

\begin{tabular}{|c|c|}
\hline Age & Number of Patients \\
\hline $15-30$ & 14 \\
\hline $31-45$ & 10 \\
\hline $46-60$ & 06 \\
\hline
\end{tabular}

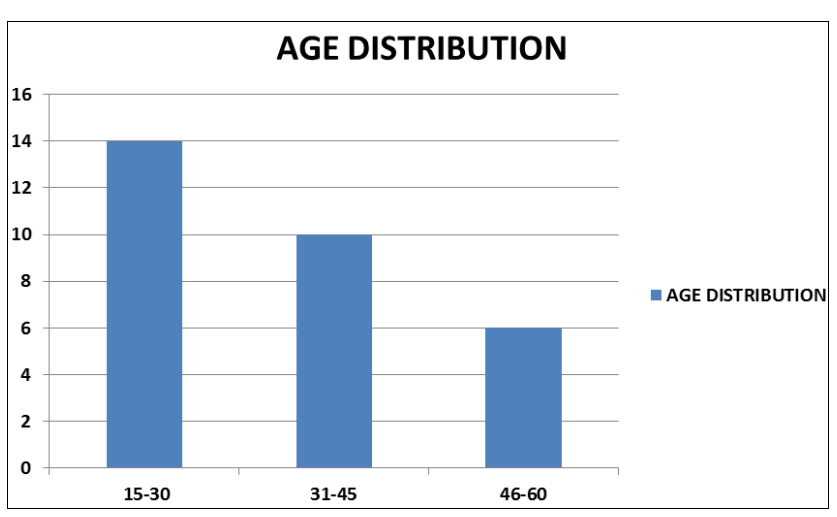

Fig 1: Bar chart showing age distribution

Table 2: Sex distribution of patients

\begin{tabular}{|c|c|}
\hline Sex & Number of patients \\
\hline Male & 22 \\
\hline Female & 08 \\
\hline
\end{tabular}




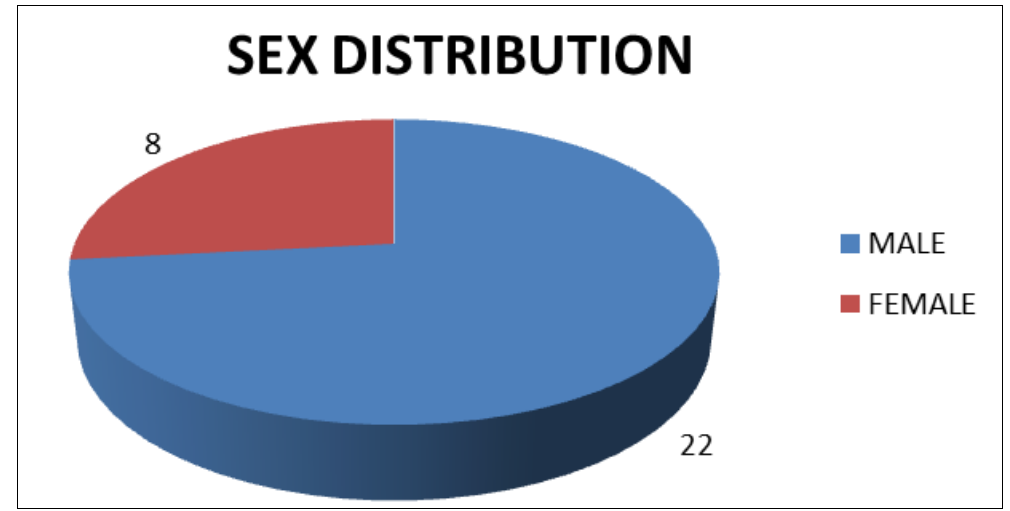

Fig 2: Pie diagram showing sex distribution

Table 3: Associated inuries

\begin{tabular}{|c|c|}
\hline Associated injuries & Number of patients \\
\hline Malleolus fracture & 06 (4 medial malleolus and 2 bimalleolar) \\
\hline Calcaneal fracture & 02 \\
\hline Tibial shaft fracture & 02 \\
\hline
\end{tabular}

Table 4: Hawkins classification

\begin{tabular}{|c|c|}
\hline Type & Number of Patients \\
\hline I & 06 \\
\hline II & 12 \\
\hline III & 08 \\
\hline IV & 04 \\
\hline
\end{tabular}

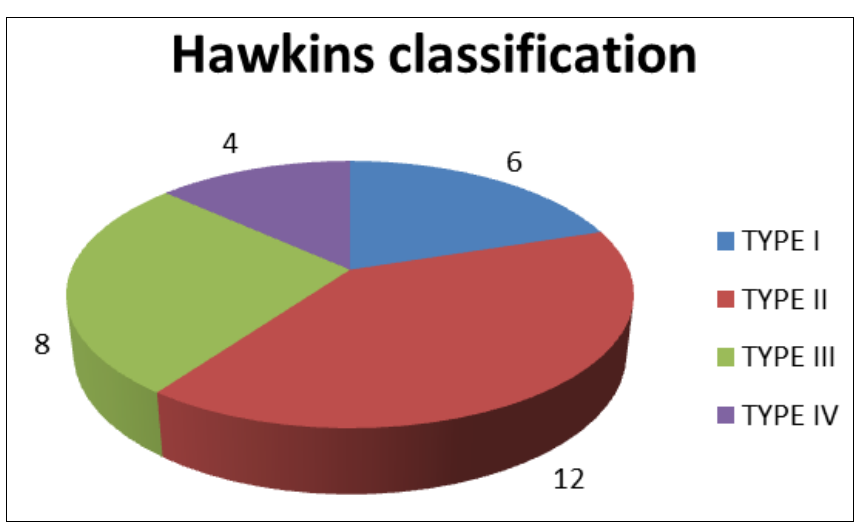

Fig 3: Pie diagram showing hawkins classification

Table 5: Surgical approach

\begin{tabular}{|c|c|}
\hline Approach & Number of Patients \\
\hline Anteromedial & 12 \\
\hline Anterolateral & 14 \\
\hline Anterior & 02 \\
\hline $\begin{array}{c}\text { Dual approach (anteromedial and } \\
\text { Anterolateral) }\end{array}$ & 02 \\
\hline
\end{tabular}

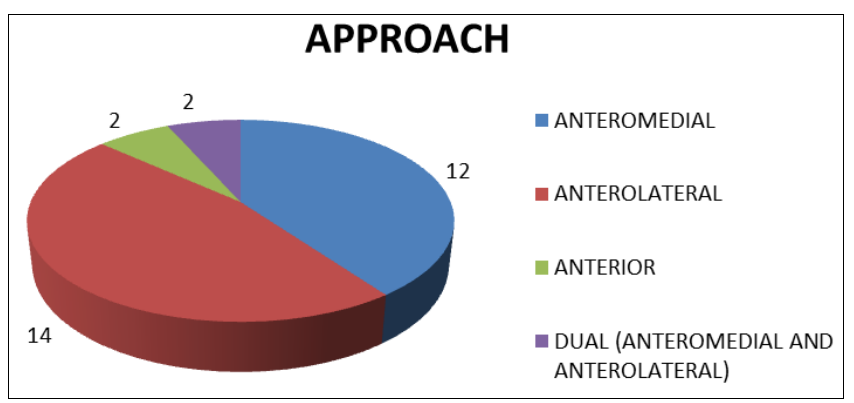

Fig 4: Pie chart showing distribution of surgical approach
Table 6: Time elapsed from injury to surgery

\begin{tabular}{|c|c|}
\hline Number of days since injury & Number of patients \\
\hline $0-10$ & 22 \\
\hline $11-20$ & 04 \\
\hline$>20$ & 04 \\
\hline
\end{tabular}

Table 7: Baird and jackson scoring system results

\begin{tabular}{|c|c|}
\hline Outcome & Number of patients \\
\hline Excellent & 00 \\
\hline Good & 02 \\
\hline Fair & 02 \\
\hline Poor & 26 \\
\hline
\end{tabular}

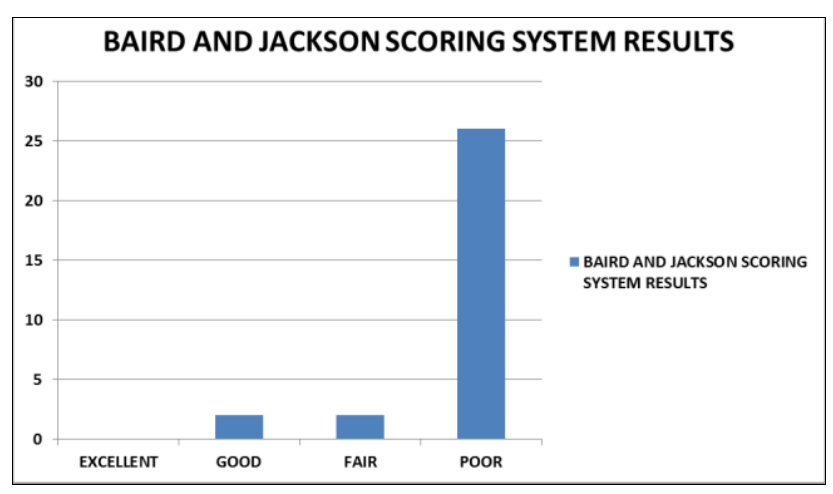

Fig 5: Bar chart showing baird and jackson scoring system results

Table 8: Aofas ankle - hind foot score results

\begin{tabular}{|c|c|}
\hline Outcome & Number of patients \\
\hline Excellent & 02 \\
\hline Good & 06 \\
\hline Fair & 12 \\
\hline Poor & 10 \\
\hline
\end{tabular}

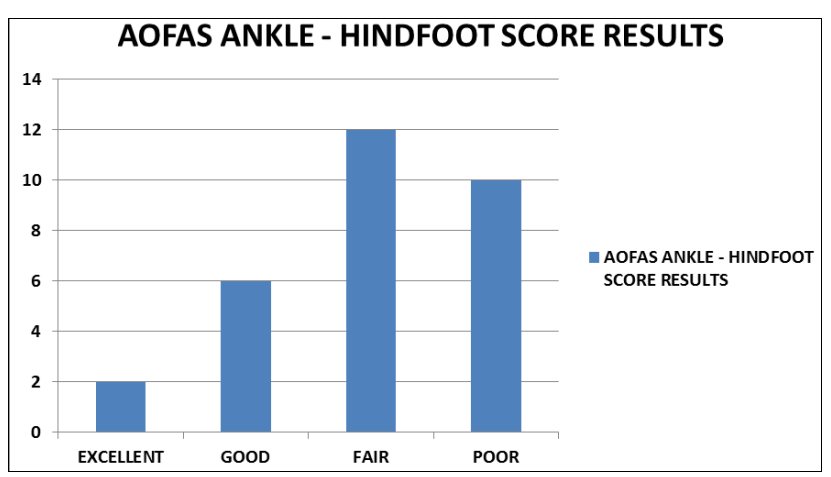

Fig 6: Bar chart showing aofas scoring results 
Table 9: Olerud and molander scoring results

\begin{tabular}{|c|c|}
\hline Outcome & Number of patients \\
\hline Excellent & 00 \\
\hline Good & 08 \\
\hline Fair & 18 \\
\hline Poor & 04 \\
\hline
\end{tabular}

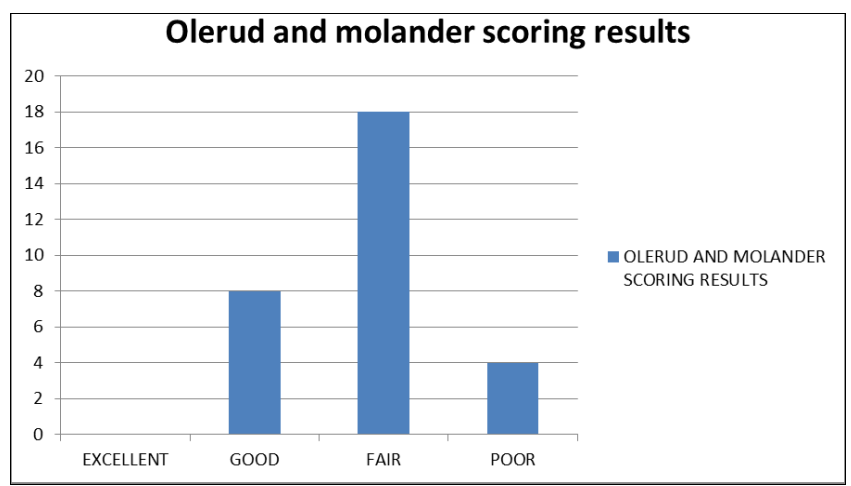

Fig 7: Bar chart showing olerud and molander scoring results

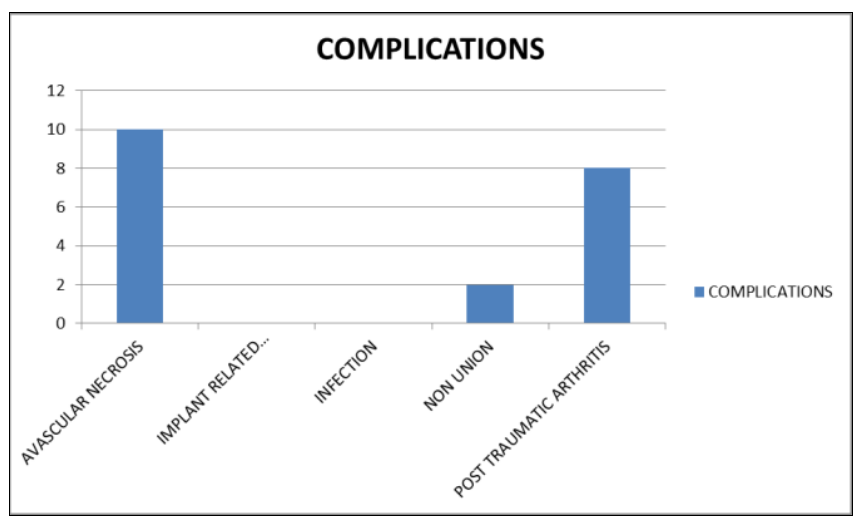

Fig 8: Bar chart showing complications
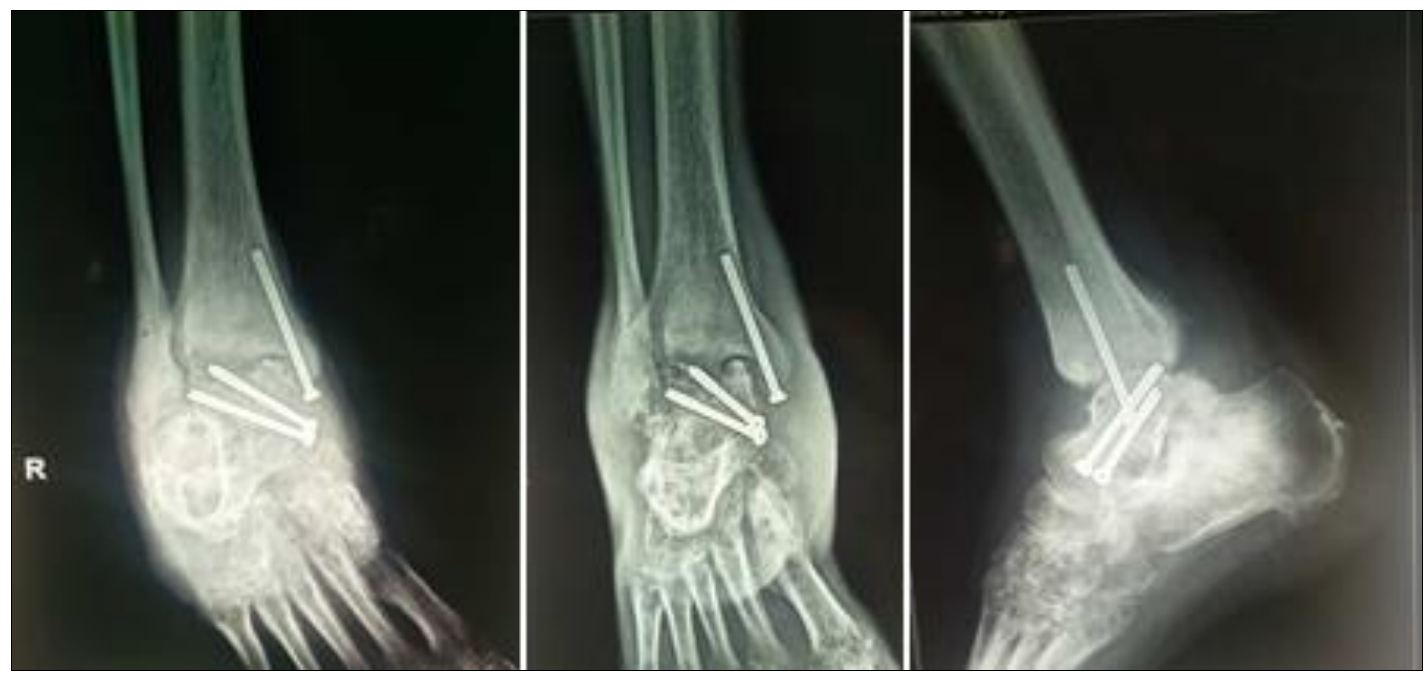

Fig 11: 6th month follow up x-rays

\section{Case no 1}

Fall Injury,

Type IV talus neck fracture

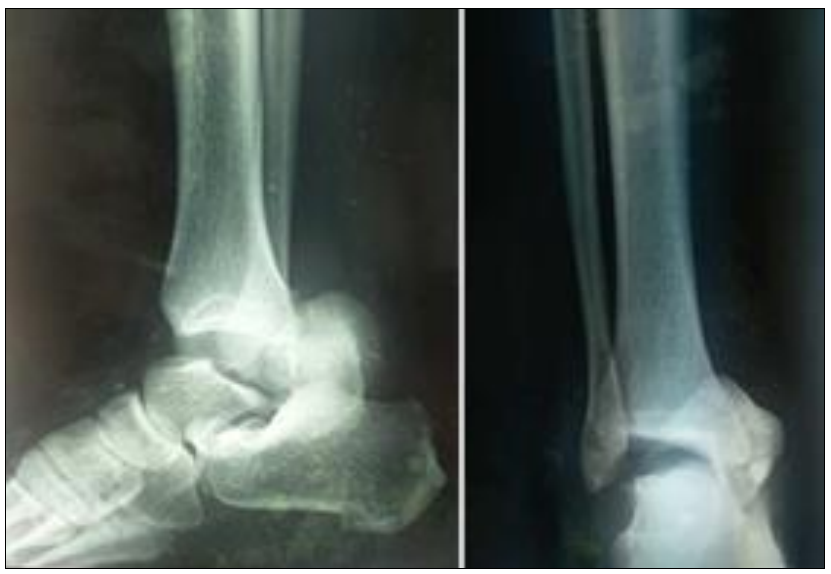

Fig 9: Preop x-rays
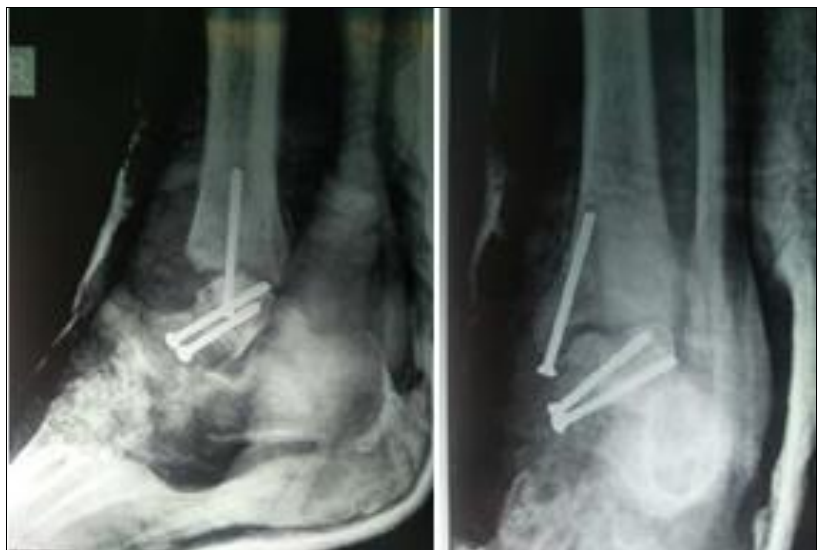

Fig 10: Post op x-rays 
Case no 2

Fall Injury,

Type IV talus neck fracture

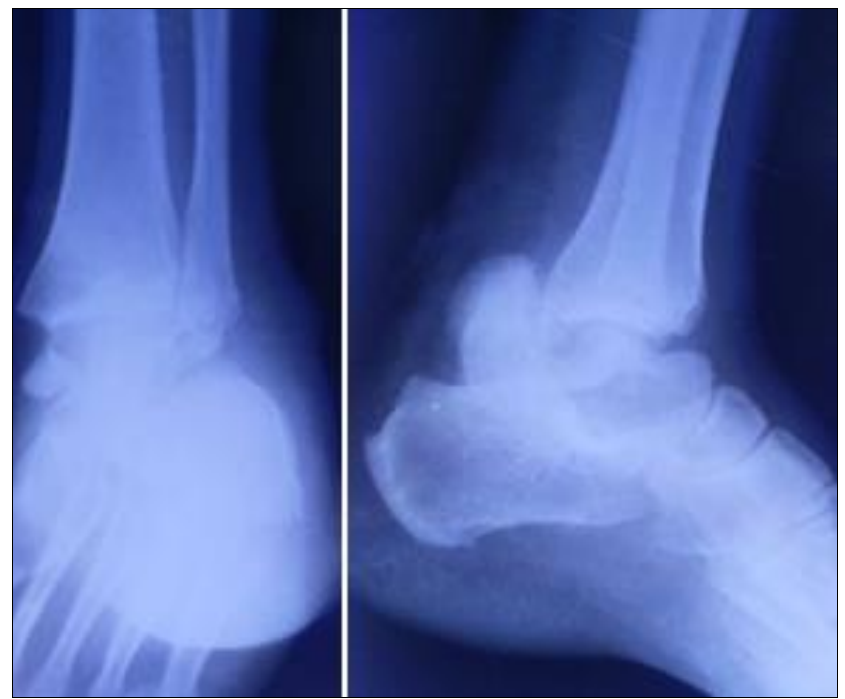

Fig 11: Preop x-rays

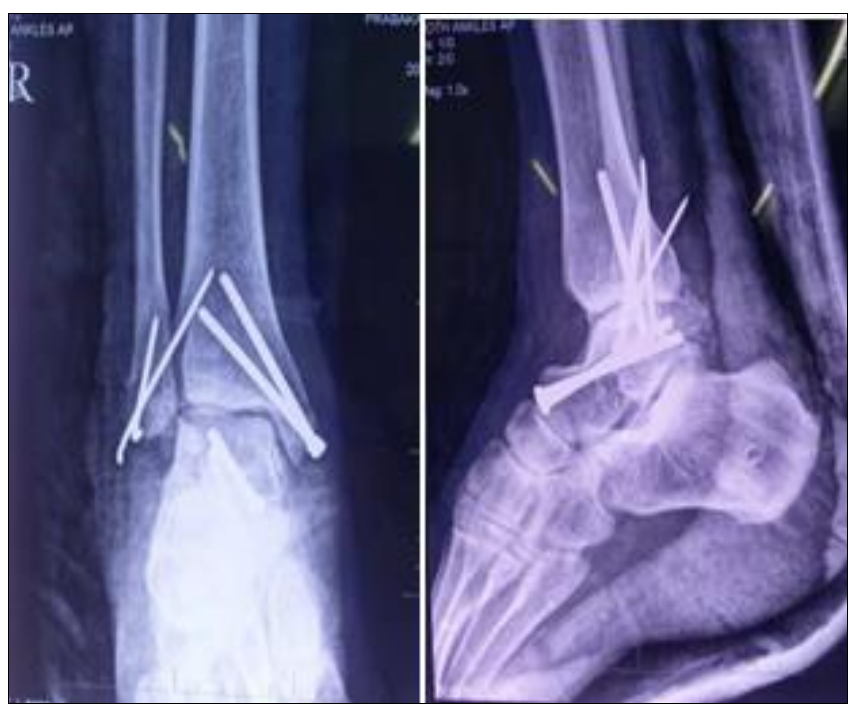

Fig 11: Immediate postop x-rays

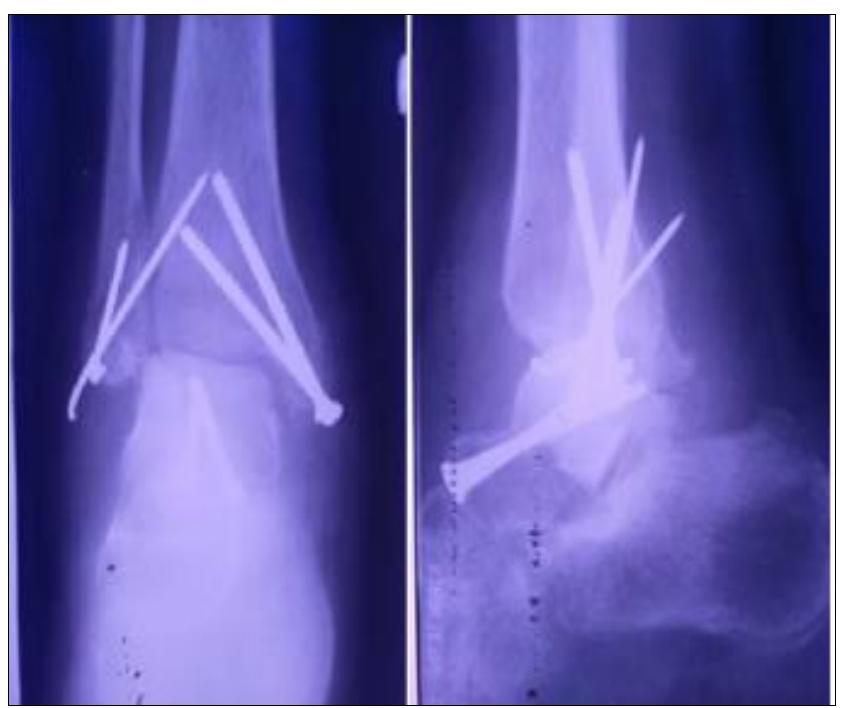

Fig 12: 6th month follow up x-ray
Case no 3

Road trafic accident, Type III talus neck fracture

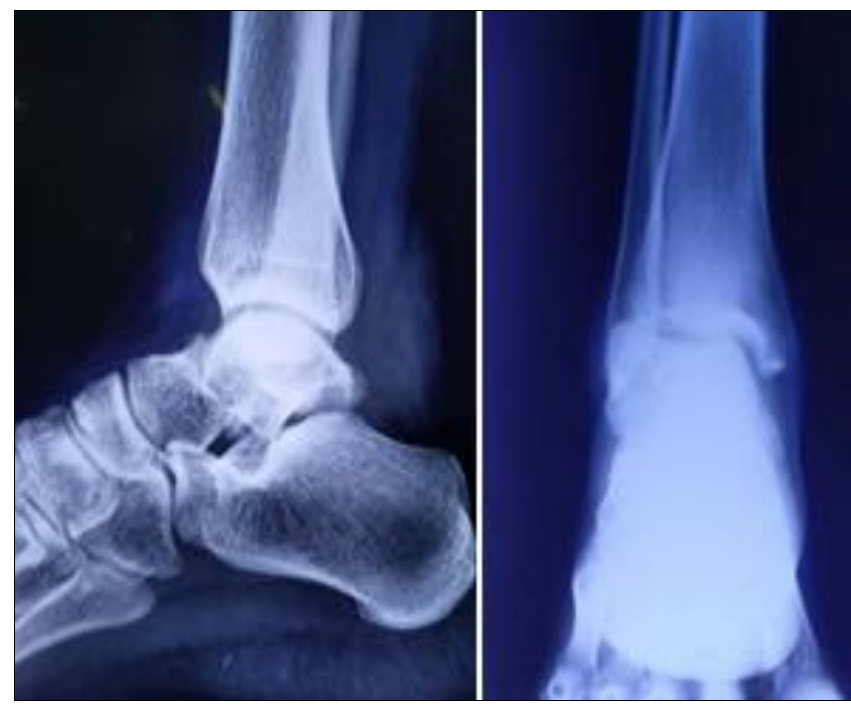

Fig 13: Preop x-rays

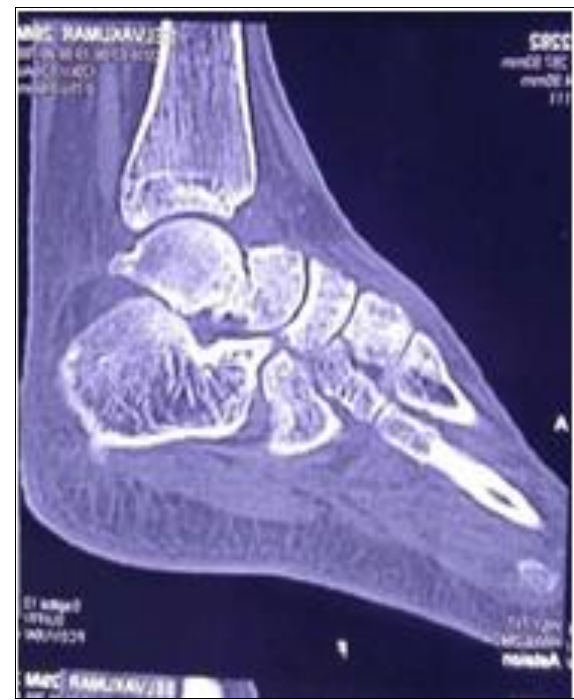

Fig 14: Preop CT scan

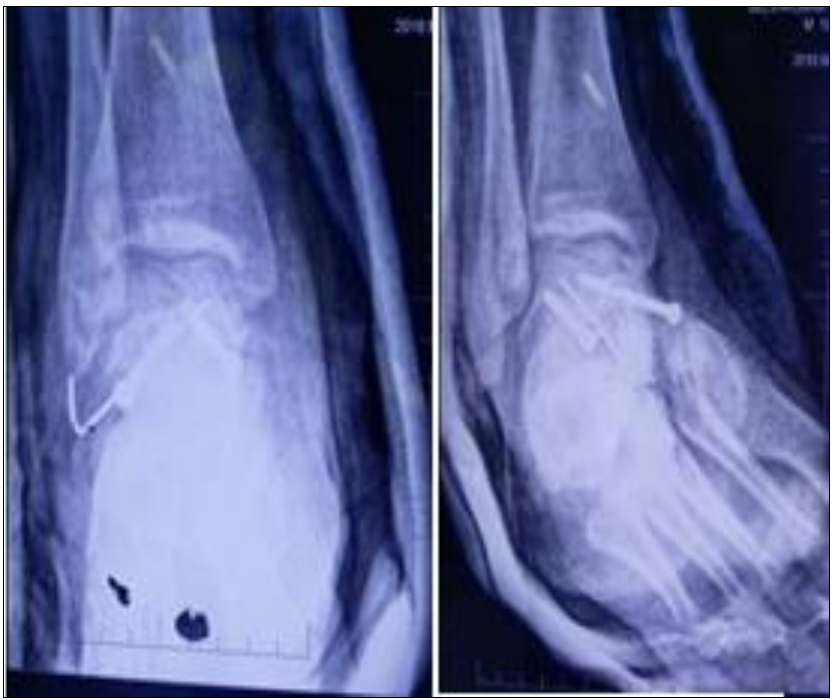

Fig 15: Immediate post op x-rays 


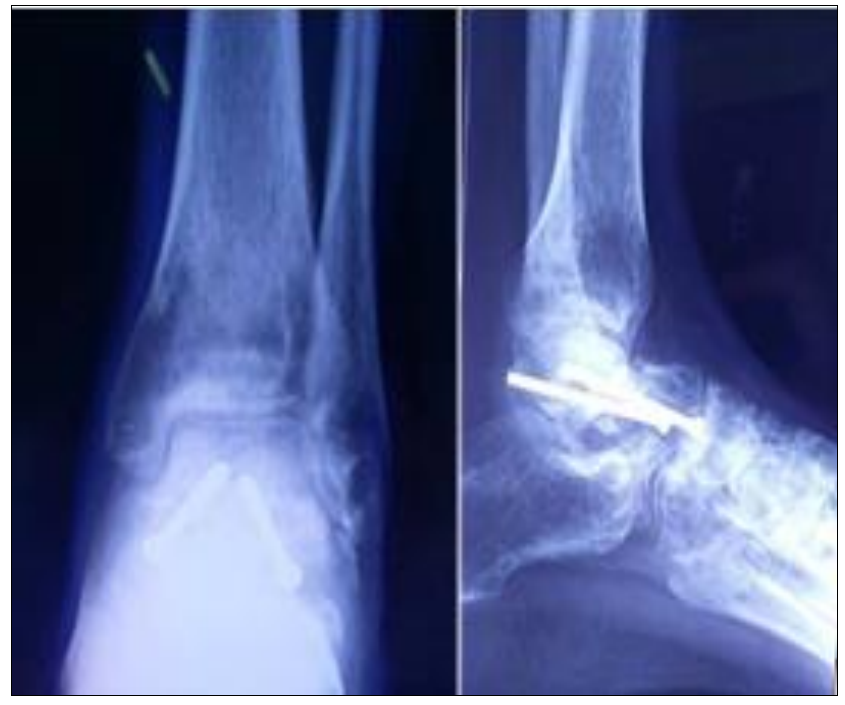

Fig 16: 3rd month follow up x-rays

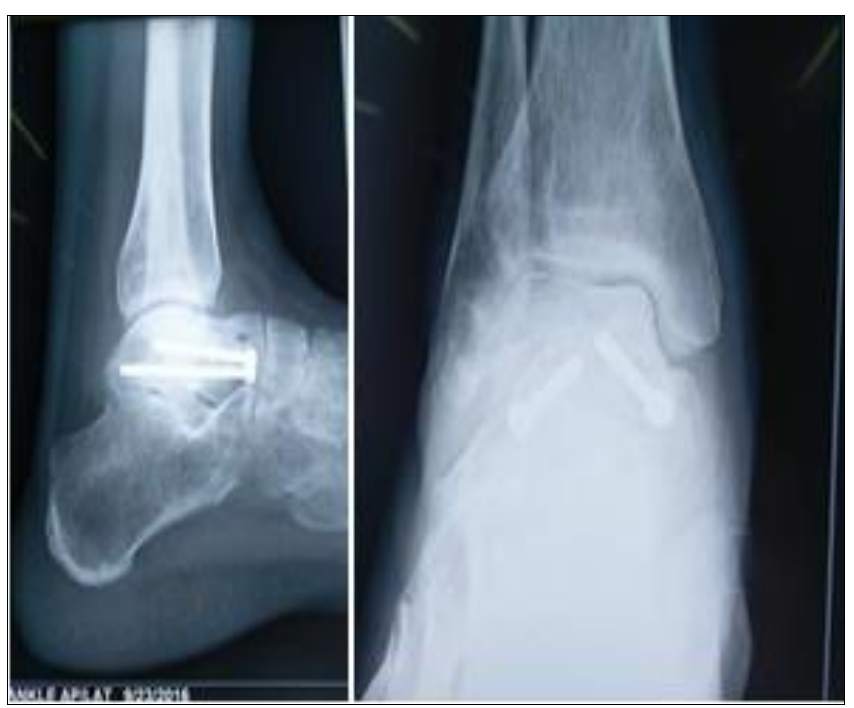

Fig 17: 6th month follow up x-rays

Case no 1

Fall Injury, Type II talus neck fracture

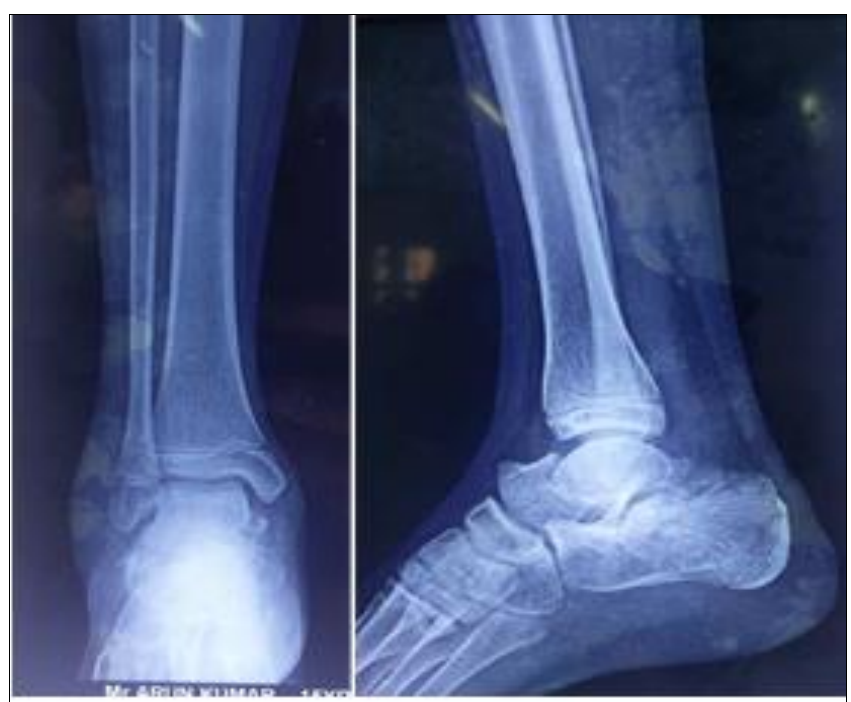

Fig 18: Preop x-rays

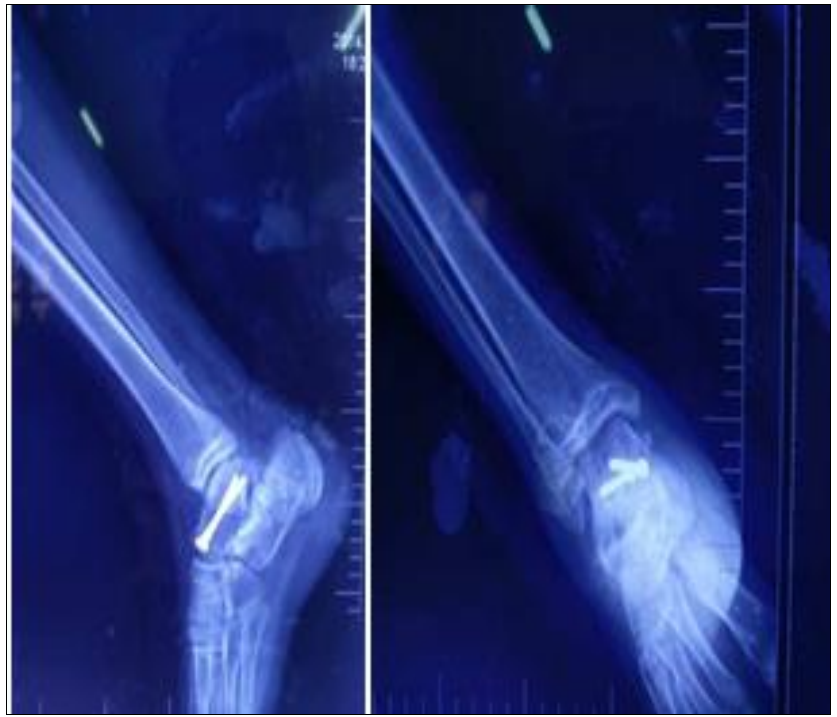

Fig 19: Immediate postop x-rays

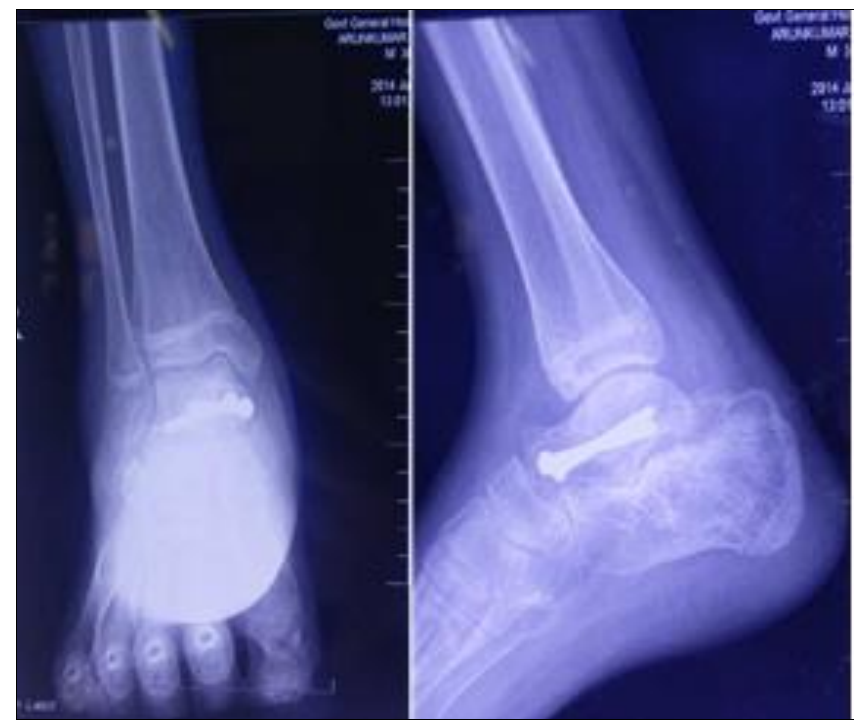

Fig 20: 3rd month follow up x-rays

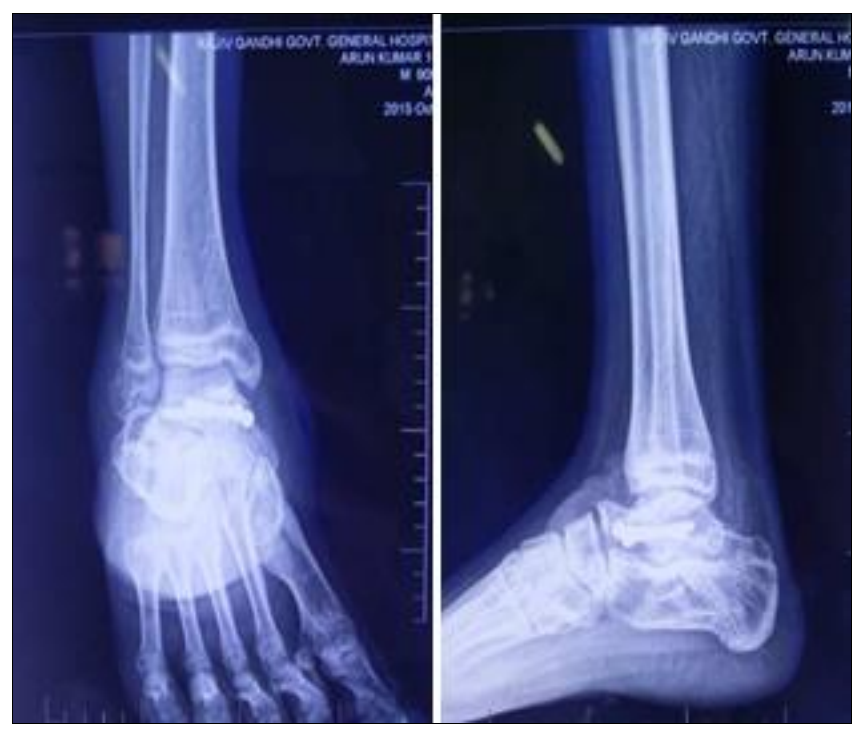

Fig 21: 6th month follow up x-rays 


\section{Discussion}

The management of these fractures is complex and there is a high complication rate. Undisplaced talar neck or body fractures are treated conservatively in most cases with very good results. However, for displaced fractures, open reduction and internal fixation is the rule. In this study of displaced talar fractures, we preferred a fast and slightly aggressive operative treatment to avoid wound complications and avascular necrosis. However, we noted a high rate of surgical failure only $30 \%$ anatomical reduction. For a better initial reduction, some authors recommend a dual anteromedial and anterolateral approach. This dual approach is sometimes associated with a medial malleolar osteotomy. This technique permits good visualization of the talus but increases the risk of skin necrosis or infection (10-20\% depending on authors) and increases the duration of surgery. Avascular necrosis is a common complication after talar fractures, the initial degree of fracture displacement is an important risk factor for osteonecrosis. The surgical delay also seems important and most authors recommend urgent reduction and stabilization of displaced talar fractures The majority of the talar surface is articular cartilage. This explains the high risk of osteoarthritis after a talar fracture. Many authors have demonstrated a relation between hindfoot misalignment or osteonecrosis and osteoarthritis. However, arthritis of the ankle and subtalar joint can occur in the absence of osteonecrosis or joint incongruity. Chondral damage can result only from the initial injury or from prolonged immobilization. At long-term follow-up, we observed a very high rate of post-traumatic osteoarthritis. This arthritis mainly affected the tibiotalar joint and the subtalar joint. Postoperative MRI scan, though gives a better picture about the joint congruency and Avascular Necrosis, it couldn't be done owing to the stainless steel screw implant involved. Though posterior to anterior screw fixation has shown to yield good results according to studies, it was not done in our study due to the technical difficulty involved. In our case, a single case was done using both anteromedial and anterolateral approach (Dual) approach. Even though the delay, in that case, was there for around 3 weeks, the outcome was excellent probably due to better visualization of the fracture and thus better reduction. The risk factor associated with the dual approach is a skin infection and necrosis, however, in young active patients occurrence of infection carries a lesser probability. The anterior approach is an alternative to the more commonly described and utilized anterolateral and anteromedial approaches. The Most commonly anterior approach is performed for talar dome fractures but the literature evidence is stating that visualization of talar neck was also excellent in the anterior approach (Cadaveric study) In our study a single case was done using the anterior approach. The case was followed up after 2 years and the outcome was found to be excellent in AOFAS and was good in Baird Jackson and Olerud Molander scoring. In the cadaveric study performed earlier, it was inferred that the talar surface area visible using the anterior approach is significantly greater than that visible using the anterolateral approach or anteromedial, without and with medial malleolar osteotomy, as well as combination approaches.

\section{Conclusion}

The study aims to analyze the outcome of closed talar fractures treated by open reduction and internal fixation and also to analyze and equate the factors which can modify the outcome. Open reduction and internal fixation are recommended for the treatment of displaced talar neck and/or body fractures. A delay in surgical fixation does not appear to affect the outcome, union, or prevalence of osteonecrosis, rather the type of fractures that might be indirectly influenced by the mechanism of injury provides a statistical significance. Road traffic accidents rather than fall from height cause a more displaced fractures and these type IV fractures though fixed with screws carries a high risk of Avascular necrosis and post-traumatic stiffness and osteoarthritis overall resulting in a poor outcome consequently.

Partially threaded cancellous screws were found to be an ideal implant of choice and both anterolateral and anteromedial approaches provided better results with the Dual approach and anterior approach providing good results. However, the case with good results was too small (1 in each) to provide a significant value. Serial X-rays need to be taken during follow up and Xray plays a major role in deciding the weight-bearing status. In patients where no signs of the union were present, the patient was found to be partial weight bearing which was not advised and further immobilization was administered. Displaced talar fractures remain a therapeutic challenge for orthopaedic surgeons. According to the literature, these fractures are often associated with a high complication rate, including malunion, osteonecrosis, or osteoarthritis The operative treatment of such fractures seems to require a balance between an aggressive treatment with a strictly anatomical reduction and essential respect of soft tissues to limit skin complications or osteonecrosis.

\section{References}

1. Abrahams TG, Gallup L, Avery FL. Nondisplaced shearing-type talar body fractures. Ann Emerg Med 1994;23(4):891-893.

doi: 10.1016/S0196-0644(94)70331-0.

2. Alvarez RG. Talar neck fractures. In: Myerson M, editor. Current therapy in foot and ankle surgery. St. Louis: Mosby, 1993, 243-248.

3. Anderson, Boyd HB, Knight RA. Fractures of the astragalus. South Med J 1942;35:160-167.

4. Fractures of the talus. In: Rockwood and Green's Fractures in Adults. Epidemiology 5th ed. Direct Plate Fixation Philadelphiapost operative management and complications, PA: Lippincott, Williams \& Wilkins; 2001.

5. Coltart WD. Aviator's astragalus. J Bone Joint Surg Br. 1952;34:545-566.

6. Blood supply of the talus comes from three arteries. These form extra osseous circulation as described by Wildenaur around the talar neck and sinus tarsi1. Elgafy H, Ebraheim NA, Tile M, Stephen D, Kase J. Fractures of the talus: experience of two level 1 trauma centers. Foot Ankle Int 2000;21:1023-1029.

7. Peterson and Goldie (Acta Orthop Scand 1974;45:260270)$,$] The cause of injury was most frequently motor$ vehicle accidents (26) and falls from heights (11)

8. Frawley PA, Hart JA, Young DA. Treatment outcome of major fractures of the talus. Foot Ankle Int 1995;16:339345.

9. Grob D, Simpson LA, Weber BG, Bray T. Operative treatment of displaced talus fractures. ClinOrthop. 1985;199:88-96.

10. Haliburton RA, Sullivan CR, Kelly PJ, Peterson LF. The extraosseous and intra-osseous blood supply of the talus. J Bone Joint Surg Am 1958;40(5):1115-1120.

11. Inokuchi $\mathrm{S}$, Ogawa $\mathrm{K}$, Usami N. Classification of 
fractures of the talus: clear differentiation between neck and body fractures. Foot Ankle Int 1996;17(12):748-750.

12. Kenwright J, Taylor RG. Major injuries of the talus. J Bone Joint Surg Br.1970;52:36-48.

13. Kitaoka HB, Alexander IJ, Adelaar RS, Nunley JA, Myerson MS, Sanders M. Clinical rating systems for the ankle-hindfoot, midfoot, hallux, and lesser toes.

14. Lindvall E, Haidukewych G, DiPasquale T, Herscovici $\mathrm{D}, \mathrm{Jr}$, Sanders R. Open reduction and stable fixation of isolated, displaced talar neck and body fractures.

15. Metzger MJ, Levin JS, Clancy JT. Talar neck fractures and rates of avascular necrosis.

16. Sanders R. Fractures and fracture: dislocations of the talus. In: Coughlin MJ, Mann RA, Saltzman CL, editors. Surgery of the foot and ankle. 8. Philadelphia: Mosby Elsevier 2007, 2076-2132.

17. Sarrafian SK. Anatomy of the foot and ankle: descriptive, topographic, and functional. Philadelphia: Lippincott, 1983, 400-407.

18. Sneppen O, Christensen SB, Krogsoe O, Lorentzen J. Fracture of the body of the talus. Acta Orthop Scand 1977;48:317-324.

19. Thordarson DB. Talar body fractures. OrthopClin North Am. 2001;32(1):65-77. doi: 10.1016/S00305898(05)70194-2.

20. Vallier HA, Nork SE, Benirschke SK, Sangeorzan BJ. Surgical treatment of talar body fractures. J Bone Joint Surg Am 2003;85(9):1716-1724.

21. Hawkins LG. Fractures of the neck of the talus. J Bone Joint Surg (Am) 1970;52:991-1002.

22. Higgins TF, Baumgaertner MR. Diagnosis and treatment of fractures of the talus: a comprehensive review of the literature. Foot Ankle Int 1999;20:595-605.

23. Szyszkowitz R, Reschauer R, Seggl W. Eighty-five talus fractures treated by ORIF with five to eight years of follow-up study of 69 patients. Clin Orthop Relat Res 1985;199:97-107.

24. Fortin PT, Balazsy JE. Talus fractures: evaluation and treatment. J Am AcadOrthop Surg. 2001;9:114-127.

25. Juliano PJ, Dabbah M, Harris TG. Talar neck fractures. Foot Ankle Clin 2004;9:723-736. doi: 10.1016/j.fcl.2004.07.001.

26. Canale ST, Kelly FB. Jr Fractures of the neck of the talus. Longterm evaluation of seventy-one cases. J Bone Joint Surg (Am) 1978;60:143-156. 